\title{
LOS EJES DE LA TERCERA VÍA EN AMÉRICA LATINA
}

\section{Roberto Patricio Korzeniewicz* y William C. Smith**}

Fecha de recepción: abril 2000

Fecha de aceptación y versión final: septiembre 2000

\begin{abstract}
Resumen: Este artículo analiza los desafíos actuales para el desarrollo de una Tercera Vía de corte social democrático en América Latina. Una vez revisadas las diferentes perspectivas y criticas en torno a dicho proyecto, este trabajo especula sobre su proceso de implementación y las posibilidades que el mismo brinda para la formación tanto de un nuevo Régimen Internacional de Política (RIP) como de un nuevo Sistema Global de Bienestar (SGB). El argumento principal es que a pesar del impacto significativo que los aggiornados proyectos social democráticos tendrían sobre la formación de un RIP en América Latina, el protagonismo y relevancia social y político de la Tercera Vía serían menores sin la simultánea articulación de un SGB, algo que los autores estiman como poco probable. El artículo concluye con una discusión sobre los posibles escenarios alternativos para la Tercera Vía y los procesos de transformación social y política en América Latina.
\end{abstract}

Palabras clave: Tercera Vía, Democracia, Desigualdad, Estado.

\begin{abstract}
This article analyzes current efforts to develop a progressive "Third Way" to social and political reform in Latin America. After briefly reviewing the perspectives of advocates and critics of such a political strategy, the article distinguishes the likely future of such a strategy in shaping the emergence of (1) a new "International Policy Regime" (IPR); and (2) a "Global Welfare System" (GWS). The article argues that although "Third Way" projects are likely to have a significant impact in shaping a new IPR in Latin America, they are less likely to result in the development of a GWS. Without the latter, the greatest political impact of a "Third Way" is likely to be restricted primarily to high income countries. We conclude by discussing alternative scenarios for "Third Way" reform and the implications of these projects for contemporary patterns of social and political change in Latin America.
\end{abstract}

Key words: Third Way, Democracy, Inequality, State.

\section{INTRODUCCIÓN}

En el nuevo milenio, diversos actores políticos, en el mundo y en Latinoamérica, se encuentran replanteando el futuro de la democracia en un mundo globalizado. Los planteos de estos actores políticos -no sólo partidos y sindicatos, sino también organizaciones no-gubernamentales (ONGs) y nuevos movimientos sociales (por ejemplo, de género o medioambientalistas) - son diferentes y muy a menudo hasta contradictorios. A veces, estos planteos acompañan nuevas convergencias entre los actores en cuestión; sin embargo, con mayor frecuencia, aún con una visión común acerca de los objetivos a alcanzar, estos planteos acarrean intensos debates acerca de los caminos a recorrer. Por ejemplo, encontramos posturas bastante divergentes con respecto a cuál debe ser el equilibrio óptimo entre regulación y mercados; en torno a los mecanismos más apropiados para generar mayor equidad; o en referencia a la relación más apropiada entre instancias nacionales o internacionales de gestión política y/o social.

Uno de los temas más críticos en estos debates ha sido el concepto de la "tercera vía". Para algunos, tal vía representa un futuro promisorio para una nueva socialdemocra- cia. Para otros, tal planteamiento representa un abandono de los ideales tradicionales de las fuerzas progresistas en favor de la incorporación de políticas de mercado y, lisa y llanamente, la aceptación de la nueva hegemonía de la ideología neoliberal. Así, mientras que para algunos la tercera vía ofrece oportunidades para fortalecer nuevos actores postergados o previamente excluidos de la comunidad, para otros, tal vía socavaría a actores políticos que fueron de gran importancia en el apoyo histórico a la socialdemocracia. Esta diversidad de interpretaciones subraya el hecho que estos debates acarrean quiebres profundos en torno a las posibilidades y restricciones que acompañan a los procesos de globalización.

En este trabajo buscamos aclarar algunos términos de estos debates. Nuestro punto principal es que debemos distinguir analíticamente dos ejes de cambio, cada uno de los cuales se caracteriza por mayor o menor protagonismo de diferentes actores políticos. Un primer eje involucra el surgimiento de lo que llamamos un "régimen internacional de

* Universidad de Maryland (EE.UU.) $\triangle$ rk81@umail.umd.ed Universidad de Miami (EE.UU.) $₫$ wsmith@sis.miami.edu 
política" (RIP). El segundo, sugiere la emergencia de un "sistema global de bienestar" (SGB). En la primera parte del nuestro ensayo analizamos cada uno de estos ejes. Luego exploramos los posibles rumbos de transformación, así como las posibles áreas de consenso y conflicto entre los actores con respecto a cada uno de estos ejes de cambio.

\section{HACIA UNA TERCERA VÍA: LAS DOS VISIONES}

En los últimos años, la posibilidad de una tercera vía ha sido fuente de intenso debate, principalmente entre los partidos socialdemócratas de Europa occidental y, en menor medida, entre los sectores centristas del partido Demócrata estadounidense y algunos grupos más moderados de la izquierda latinoamericana'. Lógicamente, hay una variación considerable con respecto a las definiciones ideológicas y las políticas específicas entre los participantes de este debate. Sin embargo, más allá de las divergencias, el modelo de una tercera vía se ha planteado en dos variantes. Primero, a partir de trayectorias nacionales específicas, se delinea una serie de orientaciones generales que, precisamente por su relativa vaguedad, permitirían la incorporación, dentro de muy diferentes contextos nacionales, de un marco "...de formulación e implementación de políticas (policy-making) que adapte a la socialdemocracia a un mundo que ha cambiado fundamentalmente en las últimas dos o tres décadas..." (Giddens, 1998: 26). Segundo, se plantea la necesidad de buscar una tercera vía de desarrollo a nivel global, a través de innovaciones profundas en la organización de mercados y regulación a nivel mundial. Estas dos variantes del modelo de la tercera vía obviamente se superponen en ciertas áreas, pero constituyen dos proyectos con dinámicas distintas. En la primera parte de este trabajo analizamos en mayor detalle estas dos variantes.

\section{La Tercera Vía como régimen internacional de política}

En términos generales, Tony Blair caracteriza a la tercera vía como "...la mejor etiqueta para la nueva política que la centro-izquierda progresista está forjando en Gran Bretaña y más allá..." (Klein y Rafferty, 1999), mientras que el sociólogo Anthony Giddens (1998: 26), uno de los más destacados intelectuales vinculados al nuevo laborismo inglés, nos dice que la "tercera vía" se refiere a un "...esfuerzo de trascender tanto la socialdemocracia de viejo estilo como el neoliberalismo..." ${ }^{2}$. Entre las características clave de la socialdemocracia de viejo estilo generalmente se incluyen el crecimiento extendido del estado de bienestar, la regulación keynesiana de los mercados, la fuerte presencia de empresas públicas en sectores estratégicos, el predominio de estrategias de crecimiento interno que desconfían de los mercados mundiales, la organización de mercados laborales en torno a la meta de pleno empleo y una división de tareas domésticas y pagas entre mujeres y hombres, y la subvalorización del medioambiente ${ }^{3}$.

Frente a estas características, la tercera vía apunta a una profunda renovación ${ }^{4}$. Para comenzar, sugiere replantear al estado, transfiriendo algunas de sus responsabilida- des a agencias ubicadas "por encima" del estado nacional (por ejemplo, al Banco Mundial, la Organización Mundial de Comercio o a los esquemas de integración regional y tratados de libre comercio) o "por debajo" (por ejemplo, municipalidades u organizaciones no gubernamentales, las ONGs); creando modalidades innovadoras para la provisión de servicios y bienes públicos; incrementando la transparencia del estado (atacando y previniendo la corrupción); mejorando la eficiencia administrativa del estado (particularmente con respecto a áreas clave de tensión social, como en lo referente al crimen, $o$ a las tensiones entre familia y trabajo); y creando nuevas formas de profundizar los procesos de democratización.

Implícito en muchas de las reformas propuestas, se plantea la necesidad de reevaluar la relación entre seguridad y riesgo. Desde este punto de vista, la tercera vía abandonaría la simple protección de los ciudadanos frente a la incertidumbre (por ejemplo, frente al desempleo) y en cambio buscaría mejorar la capacidad del estado de preparar a los ciudadanos para enfrentar exitosamente situaciones de riesgo y la vulnerabilidad implícitas en una sociedad de mercado. Igualmente implícita está la idea de una reevaluación del potencial de los mecanismos de mercado para optimizar el uso de recursos, la distribución de la información y el crecimiento económico en general. En síntesis, la tercera vía se desarrollaría en un estado de inversión social, de profunda democratización y de integración plena en los procesos de globalización, generando una dinámica en la cual cada una de estas dimensiones se fortalecería en un círculo virtuoso. Tales dinámicas suponen también el establecimiento de un amplio compromiso social entre ricos y pobres, pero sin el predominio del viejo estilo neo-corporativista de concertación social entre capital, trabajo y estado que anteriormente habría caracterizado a la socialdemocracia clásica.

El surgimiento de la tercera vía se atribuye usualmente a un cuestionamiento profundo de los mecanismos prevalecientes de regulación estatal de los mercados. La crisis de estos mecanismos, manifestada a través de las bajas tasas de crecimiento económico después de la década de los sesenta, lleva a la necesidad de reevaluar "la teoría económica del socialismo," ya que "...siempre fue inadecuada, subestimando la capacidad del capitalismo de innovar, adaptarse y generar creciente productividad..." (Giddens, 1998: 4). La

1. Para un análisis sobre los partidos socialdemócrata en diferentes países de la Unión Europea, pueden consultarse SFERZA (1999); MERKEL (1999); El País (1999f y 1999g); DAHRENDORF (1999). Con respecto a la vaguedad de estos planteos, véase una discusión online muy interesante entre participantes norteamericanos e ingleses sobre la tercera vía. Los participantes destacaron no sólo la vaguedad, diferencias terminológicas y cambios históricos del contenido de la tercera vía, sino también su carácter "vagamente místico" (HALPERN Y MiKOSZ, 1998).

2. El Presidente de Estados Unidos, William Clinton, ha adoptado un lenguaje similar al de Tony Blair, indicando que “...nos estamos moviendo mas allá del debate estéril entre aquellos que dicen que el estado (government) es el enemigo y los que dicen que el estado es la solución. Mis conciudadanos americanos, hemos encontrado una tercera vía..." (citado en KLEIN y RAFFERTY, 1999).

3. Para descripciones y análisis de la socialdemocracia clásica, ver PRZEWORSKI (1985) y OFFE (1984).

4. En este párrafo resumimos las ideas presentadas por GIDDENS (1998). También véase el más reciente libro de GidDENs (2000) donde responde a sus críticos. 
crisis del estado benefactor y de la propia teoría keynesiana que sustentaba a la socialdemocracia (ver Przeworski, 1985), habría sido precipitada por los procesos de globalización a partir de la década del setenta, y por el concomitante auge del neoliberalismo durante la década del ochenta. Frente al desafío de los programas neoliberales de derecha, la tercera vía se plantea como una renovación de los ideales del socialismo y de la socialdemocracia.

Así, América Latina es lugar común para rastrear tal cuestionamiento a la crisis de la "década perdida" de 1980 y su impacto sobre los mecanismos de regulación estatal y los modelos de industrialización de posguerra. En una primera instancia, este cuestionamiento se habría cristalizado en el "Consenso de Washington" el cual condujo a las medidas de ajuste estructural y reformas de mercado que caracterizaron a la región en los últimos años. Una visión optimista acerca del futuro de estos cambios enfatizaría que en la actualidad estamos presenciando el surgimiento de un nuevo "consenso" o una "segunda generación" de reformas, a través del cual ciertas instituciones e instancias supranacionales (desde el Banco Mundial a la CEPAL, incluyendo también las recientes Cumbres de las Américas) impulsan una nueva agenda de reforma social, enfatizando la necesidad de avanzar los procesos de democratización, de mejorar la transparencia y eficiencia administrativa del estado, de erradicar la pobreza y atacar la desigualdad en Latinoamérica como pasos imprescindibles para promover un mayor crecimiento económicos. Esta perspectiva optimista podría enfatizar también que el surgimiento de una tercera vía se vería fortalecida por el surgimiento o fortalecimiento de otros actores sociales y políticos - por ejemplo, nuevas demandas, formas de acción y de organización construidas en torno a la creciente participación femenina en el mercado laboral formal (con toda la redefinición de lo público y lo privado implícita en esta participación), la reevaluación del impacto del crecimiento sobre el medioambiente y/o cambios en actitudes generacionales frente a la democracia y el crecimiento económico (Giddens. 1998: 20). En este sentido, el nuevo papel de actores supranacionales en la articulación de estrategias alternativas de desarrollo “desde arriba” estaría siendo acompañado, "desde abajo" por una aparente renovación de la sociedad civil, manifestada por ejemplo en la creciente importancia de las ONGs, los movimientos sociales y otras manifestaciones de la sociedad civil ${ }^{\natural}$.

Finalmente, el surgimiento de nuevas alternativas políticas es visto también como efecto de la crisis de los modelos de desarrollo centrados en torno a la industrialización. En este sentido, una de las principales fuentes tradicionales de apoyo de las diversas versiones del "partido socialdemócrata” habría sido debilitado por la erosión de la clase obrera industrial y del sindicalismo (Giddens, 1998: 20). Desde este punto de vista, y dadas las nuevas inquietudes políticas que hemos discutido hace un momento, “...los partidos socialdemócratas ya no tienen un 'bloque de clase' consistente en que apoyarse. Ya que no pueden depender en sus identidades previas, deben crear nuevas en un ambiente social y culturalmente más diverso..." (Giddens, 1998: 23).
Cabe observar que los propios ideólogos y adherentes de la tercera vía reconocen que estos cambios en la base de apoyo social son problemáticos. Por ejemplo, Robert Reich, ex-Secretario de Trabajo en la primera administración de Bill Clinton, indica con cierta preocupación que "...la tercera vía no tiene organización de base (grassroots)..." Para Reich (1999),

“... la agenda -liberar las fuerzas del mercado mientras que se facilita la transición para aquellos que alternativamente se quedarían atrás- no tiene un núcleo natural de apoyo fuerte en el electorado. No se puede encontrar apoyo en la izquierda tradicional, para mucho de la cual sería preferible proteger y preservar la vieja economía. Y tampoco se lo puede encontrar en la derecha conservadora, mucho de la cual está floreciendo en la nueva economía y no quiere un gobierno activista sacándole su dinero..." ?.

Reich concluye sus observaciones indicando que este callejón sin salida solo podría ser resuelto con el establecimiento de un "New Deal" entre los ganadores y perdedores en las nuevas dinámicas de globalización".

En conclusión, el modelo de la tercera vía se presenta como un modelo que permitiría profundizar las prácticas democráticas y crear condiciones para un crecimiento económico sostenido acompañado, como condición, por una mayor equidad social resultante de la mayor eficiencia del estado para preparar a la ciudadanía para aprovechar las oportunidades ofrecidas por el mercado.

¿Es la tercera vía un modelo sólo relevante a los países de alto desarrollo (aunque, como destacamos anteriormente, en estos países se debate la medida en que existe un modelo común a las diversas experiencias nacionales)? $¿ \mathrm{O}$ nos encontramos ante un planteamiento relevante a Latinoamérica? Obviamente, depende de nuestro punto de partida.

Si entendemos los dilemas enfrentados por la tercera vía como específicos a ciertos partidos de centro izquierda cuyo crecimiento electoral se vio limitado a países de alto desarrollo, la relevancia de esta nueva estrategia es bastante limitada. Pero Przeworzki (en prensa) nos recuerda que a veces emergen situaciones de equilibrio en las formas de hacer política. El mismo autor introduce el término "régimen de política" (policy regime) para referirse a estos equilibrios, donde la lógica de la competencia electoral y el aprendizaje social llevan a los partidos políticos a trascender apegos ide-

5. Sobre esta nueva agenda, ver por ejemplo: BANCO MUNDIAI (1998), BID (1998 y 2000), STALLINGS y PERES (2000) y la discusión en KORZENIEIWICZ Y SMITH (2000).

6. Muy relevante al estudio de esta problemática es la contribución de proyecto del Programa de Instituciones Económicas Internacionales de FLACSO/Argentina, dirigido por Diana Tussie. Véase Tussie (2000). Sobre el papel de las ONGs y movimientos sociales en este proceso, véanse JELIN (1997 y 1998).

7. Sobre las consecuencias de esta ausencia de apoyos sociales para la implementación de políticas redistributivas, ver REICH (1997: 6), quien hace una crítica a la administración Clinton.

8. Como indicaremos más abajo, desde el punto de vista de la cre ación de un "sistema global de bienestar" (SGB) este problema es aún más serio que lo que aparenta. 
ológicos para converger en torn , a políticas similares. En este trabajo, modificamos modestamente el término utilizado por Przeworski para calificar "regímenes internacionales de política” (RIP) a la creciente convergencia -en cuanto a algunos arreglos político-institucionales y políticas macroeconómicas y sociales- que caracteriza a una amplia gama de naciones, más allá de diferenci.ıs en la arquitectura política o niveles generales de ingresos entre estos países. Por ejemplo, el modelo implícito en el estado de bienestar de posguerra puede ser considerado como constituyente de un régimen internacional fordista, cuyas características sociales y políticas específicas variaron de región a región, o entre países pobres y ricos, pero compartiendo ciertas similitudes fundamentales (por ejemplo, en la relación entre mercado y estado, o en las formas de hacer política). Desde tal punto de vista, la tercera vía puede ser considerada como un nuevo RIP emergente que podría repı esentar en el futuro cercano algo similar a lo que el estad, de bienestar representó a mediados del siglo $\mathrm{XX}^{9}$.

\section{La Tercera Vía como sistema global de bienestar}

Más allá del surgimiento de la tercera vía como un potencial RIP, algunas voces han planteado la necesidad de elaborar una tercera vía de desarrollo, que permita disminuir patrones de desigualdad entre naciones de bajos y altos ingresos, profundizar la democratización a nivel global y generar mayor bienestar ${ }^{10}$. Tal como se sugiere en Korzeniewicz y Smith (2000), estos objetivos generales se acompañan por nuevas instancias de organización, que involucran en redes a agentes colocados por encima (por ejemplo, organizaciones supranacionales, u OSNs) y por debajo (ONGs) de los estados nacionales. Para referirnos a este eje de cambio institucional, utilizamos el término "sistema global de bienestar" (SGB). Los dos ejes de la tercera vía quedarían reconfigurados por: un régimen de política internacional organizado en torno a cambios institucionales dentro del estado nacional; y un sistema global de bienestar organizado en torno a cambios institucionales en la organización de la cconomía-mundo.

Podemos ilustrar este segundo eje con un discurso reciente del Presidente Bill Clinton pronunciado en la Universidad de Chicago en 1999. El discurso comienza discutiendo el conflicto en Kosovo, pero inmediatamente pasa a evaluar la situación de la economía global, indicando que esta economía está generando ganadores pero también muchos perdedores, y que para responder a esta dinámica es necesario expandir a nivel global lo que el estado de bienestar asentó en Estados Unidos a lo largo del siglo XX. De acuerdo con el discurso, la adopción de tales medidas es inevitable, ya que

“... la comunidad global no puede existir como la 'historia de dos ciudades' una moderna e integrada con un teléfono celular en cada mano y un McDonald's en cada esquina, y la otra ahogada en la pobreza y crecientemente resentida, llena de problemas de salud pública y del medioambiente que nadie puede manejar..." (Clinton, 1999) ${ }^{11}$.
Al considerar el posible surgimiento de un SGB, se podría objetar que aún no son evidentes los arreglos institucionales globales que se necesitarían como esqueleto para tal arquitectura. No sólo las OSNs (por ejemplo, Banco Mundial o Fondo Monetario Internacional) siguen funcionando "por arriba" de los principales actores políticos y sociales a nivel nacional sino también, en forma relacionada, los diferentes contextos nacionales continúan siendo el espacio privilegiado de la política. Sin embargo, haciendo un paralelo entre el presente y el largo período que caracterizó el surgimiento del estado de bienestar durante la primera mitad del siglo XX se puede delinear una perspectiva alternativa sobre los procesos de cambio actuales. Sintetizamos, entonces, algunos de estos paralelos históricos.

Tanto entonces como ahora, la lenta emergencia de nuevos arreglos institucionales hace difícil predecir su forma eventual en medio del cambio. Así, en la década del diez o del veinte, no sólo en Latinoamérica, sino en la mayoría de los países y regiones del mundo, se hacía difícil prever que en sólo dos décadas se daría una casi total convergencia en torno a la necesidad de la regulación estatal de los mecanismos del mercado (en las décadas del cuarenta o del cincuenta se continuaría debatiendo cómo regular tales mecanismos, o con qué objetivos, pero pocos mantendrían su confianza en la suficiencia de los mercados). De la misma manera, hoy presenciamos sólo formas preliminares e inestables de los mecanismos de protección y de mediación política que prevalecerán de aquí a veinte años.

Los arreglos institucionales emergentes involucran una nueva importancia de actores ubicados por encima y por debajo de las fuerzas políticas existentes, actores articulados entre sí por objetivos clave compartidos. Recordemos que en gran parte de Latinoamérica a principios de siglo, los propios estados nacionales constituían todavía un nuevo actor que buscaba aún establecer su predominio sobre el poder de núcleos políticos establecidos a un nivel más local, y que en muchos sentidos este predominio avanzó a través de su relación con (y capacidad de interpelar a) nuevas fuerzas emergentes por debajo del poder local, principalmente manifestadas a través del movimiento obrero (aunque nuestra historiografía generalmente haya enfatizado las tensiones dadas entre estado nacional y movimiento obrero). En este marco, OSNs y diversas manifestaciones de la sociedad civil

9. En este sentido, al estado de bienestar de posguerra correspondería un RIP de fordismo global o de embedded liberalism (RugGIE, 1982). Sin embargo, no coincidimos con las posturas "hiperglobalistas" (HELD MCGREW, GOLBLATT y PERRATON, 1999), pues existe un debate considerable acerca del grado de convergencia (y resistencia) nacional generado por la aceleración de la globalización. Para una reseña de estos debates, ver KeOHANE y Milner (1996), Berger y DORE (1996), BolX (1998), GARrETt (1998) y KiTSCHELT, et al. (1999).

10. Por supuesto, como objetivos generales, tales metas también estuvieron presentes en los modelos de desarrollo de la posguerra.

11. Con un lenguaje diferente, subrayando la supremacía de la política sobre el mercado e insistiendo que apoyan "...una economía de mercado pero no una sociedad de mercado..." la reunión de noviembre de 1999 de la Internacional Socialista en Madrid llegó a conclusiones similares acerca de la necesidad de desarrollar nuevas formas de governance global (ver New York Times, 1999a y El País, 1999a, 1999b, 1999c, y 1999e). Del mismo modo, resultan llamativas las coincidencias con estos análisis en algunos de los discursos recientes de James Wolfensohn, presidente del Banco Mundial (ver Banco Mundial, 2000; Financial Times, 2000). 
-como ONGs, o los movimientos de mujeres y medioambientales- representan hoy en día las fuerzas clave de los nuevos arreglos institucionales que pasarán a predominar en las próximas décadas. Implícito en esta interpretación, vemos a los procesos de globalización no como proyecto exclusivo de elites económicas y políticas beneficiadas por la expansión de mercados, sino también como el resultado de las demandas y acciones de grupos sociales previamente excluidos de arreglos políticos y mercados.

Aunque los nuevos arreglos institucionales involucran nuevas formas de organización, de regulación de mercados y de hacer política, las fuerzas políticas tradicionales continúan predominando en la constitución de estos nuevos arreglos. Así, el populismo, como variante latinoamericana del estado de bienestar, no surgió a nivel nacional de un vacío político (aunque tal interpretación fuera frecuentemente promulgada en la historiografía o la propia autobiografía de aquel fenómeno), sino que fue muy a menudo anticipado por fenómenos similares manifestados a nivel local (por ejemplo, en ciudades y provincias argentinas durante las décadas de 1920 y de 1930, o en estados de México durante la revolución o la década de 1920). Desde tal punto de vista, argumentaríamos que las diferentes versiones de la tercera vía representan esfuerzos incipientes de definir nuevas formas de organización, de regulación de mercados y de hacer política que caracterizarán a los arreglos institucionales del siglo XXI.

Como Karl Polanyi (1947), autor de La gran transformación, sugerimos que el avance de los mercados se acompaña inevitablemente por una mayor regulación política de estos mercados, en lo que aquel autor llamó un "doble movimiento". En este marco, el capitalismo siempre ha entrañado tanto mercados como su regulación, por lo cual la constante creación de nuevos sistemas de bienestar es parte constitutiva de la economía-mundo. A comienzos del nuevo milenio debemos considerar la posibilidad de que OSNs y ONGs representen la base de un nuevo eje de cambio institucional que apunta a transformar las dinámicas de la economía-mundo para generar mayor bienestar a nivel global.

\section{Convergencias y conflictos entre los dos ejes}

Hemos planteado hasta ahora la visión optimista acerca de dos ejes posibles para la formulación de una tercera vía. Queremos enfatizar que estos ejes son distinguibles tanto desde el punto de vista de los actores que los articulan (por ejemplo, partidos políticos, empresarios y sindicatos nacionales en el caso del RIP, y OSNs y ONGs en el caso del SGB), así como de las modalidades y estrategias de acción. Por estas razones, los actores de cada eje convergen en algunas áreas de acción y reforma institucional, pero divergen en otras. Pasamos a dar algunos ejemplos.

Mientras que las alianzas populistas tradicionales del viejo modelo estado-céntrico latinoamericano (ver Cavarozzi, 1992) se derrumbaron antes de la embestida violenta de la reestructuración del mercado, la integración comercial hemisférica y la globalización, la actual renovación incipiente de las fuerzas de la "socialdemocracia" incorpora algunos remanentes de las fuerzas políticas convencionales (sindicatos, par- tidos, entes estatales nacionales y locales), junto con nuevos grupos vinculados a la creciente convergencia emergente entre las OSNs y las ONGs, actores que transforman la tradicional lógica de las alianzas sociales y políticas plausibles. Nos referiremos a esa rearticulación de fuerzas políticas como "coaliciones policéntricas de desarrollo" que se distinguen de los patrones estado-céntricos típicos de los arreglos políticos previos a los ochenta ${ }^{12}$.

Como ejemplo, podemos examinar brevemente el "Consenso de Buenos Aires”, un documento que representa a un amplio conjunto de fuerzas políticas de muchos países de América Latina (Castañeda, et al. 1998). Ideológicamente y desde el punto de vista organizacional, el "Consenso" es un proyecto que compromete a muchos de los líderes de los partidos de la "nueva izquierda" (Cuahetémoc Cárdenas del Partido de la Revolución Democrática de México; Carlos "Chacho" Álvarez del FREPASO de Argentina; Luiz Inácio Lula da Silva y Vicentinho del Partido dos Trabalhadores de Brasil y Ricardo Lagos del Partido Socialista y del Partido por la Democracia de Chile, entre otros), así como algunos intelectuales “post-marxistas" (Jorge Castañeda, Roberto Mangabeira Unger) en la construcción de un nuevo rostro para la socialdemocracia en la región. La meta es la creación de nuevas alianzas democráticas que reúnan a partidos, movimientos y líderes de centro (cuya tarea sería la de "darle expresión a la inconformidad de la clase media") y de izquierda (cuya tarea es "enfrentar la desigualdad" y "combatir la exclusión social”) con el fin de reconfigurar y compatibilizar la lógica de la globalización con el fortalecimiento de las instituciones de la democracia representativa, incorporando un amplio conjunto de actores sociales. En consecuencia, la participación y la condición de ciudadano se extienden más allá de la arena electoral, hacia el ámbito de la cultura y de la organización de la economía política (Castañeda, et al. 1998; Dagnino, 1998).

Aunque los principios generales, las críticas y denuncias respecto del neoliberalismo en el Consenso de Buenos Aires eclipsan sus propuestas de política específicas, este documento y otros manifiestos similares pueden leerse como las primeras etapas de un aggiornamiento impregnado del realismo político necesario para elaborar estrategias y políticas progresistas en un orden social emergente, inmerso en la lógica de los mercados mundiales. Este nuevo estilo de discurso socialdemócrata (aunque algunos de los participantes quizás no aceptan esta denominación) concuerda a su vez con las teorías del crecimiento endógeno y los avances recientes en el pensamiento acerca del crecimiento con equidad ${ }^{13}$. De

12. Aunque nuestra utilización del término coalición policéntrica tiene ciertas semejanzas con las nociones recientes de "redes asociativas" (Chalmers, MARTIN y PIESTER, 1997), "redes de movimientos sociales" (ALVAREZ, DAGNINO Y ESCOBAR, 1998) y "campo de acción policéntrica y heterogénea" (ALVAREZ, 1998), el énfasis que esos autores colocan habitualmente en los lazos horizontales de solidaridad y reciprocidad no excluye la posibilidad de que en el futuro puedan constituirse arreglos jerárquicos más fuertes dentro de las coaliciones policéntricas.

13. Ver KoRZENIEWICZ y SMITH (2000), donde resumimos este consenso desde el punto de vista de tres premisas básicas: 1) no hay alternativa para el crecimiento impulsado por el mercado; 2) el crecimiento y el bienestar no están en una relación de suma-cero; y 3 ) los mecanismos de mercado deben complementarse con los nuevos arreglos institucionales 
hecho, aunque su formulación precisa es bastante diferente, se observan estrechos paralelos, insospechados y sorprendentes, entre el discurso reelaborado de la izquierda de la post-Guerra Fría y los temas, estrategias y políticas abogadas por los tecnócratas de las instituciones multilaterales o supranacionales como el Banco Mundial, el BID y la CEPAL.

Además, y más allá de premisas abstractas, existe una convergencia considerable en varias áreas específicas que puede proporcionar una base fructífera para futuras reformas. Al igual que en recomendaciones de política recientes producidas por las OSN con sede en Washington, el "Consenso de Buenos Aires" enfatiza la necesidad de seguir avanzando en la integración económica regional como medio de perseguir el crecimiento económico, apoya los esfuerzos por elevar las tasas de ahorro interno y promover las inversiones productivas, y reclama que el estado incremente los recursos fiscales mediante impuestos al consumo. En este marco, la pobreza y la desigualdad son consideradas como áreas clave para la intervención de las políticas, mediante oportunidades educativas más amplias, mejor acceso al cuidado de la salud y políticas fiscales redistributivas que dirijan recursos escasos a los sectores sociales más excluidos.

El acuerdo subyacente con respecto a estos temas posee el potencial de permitir una relación de fortalecimiento mutuo entre el crecimiento, la equidad y la democracia. El crecimiento económico es necesario para proveer los recursos fiscales que financien inversiones en bienestar social. Por otra parte, además de contribuir al crecimiento, una mayor equidad es fundamental para la estabilidad social y la plena confianza en las instituciones democráticas y las reformas del mercado. El crecimiento con equidad puede a su vez ayudar a superar los resultados distributivos de suma-cero, y con ello prolongar los horizontes temporales, facilitando el aprendizaje social requerido para abandonar estrategias de confrontación y consolidar un compromiso de clase democrático ${ }^{14}$

La construcción de nuevas coaliciones policéntricas de orientación socialdemócrata se ve condicionada por disputas importantes. En primer lugar, hay desacuerdo en cuanto al alcance óptimo de la reforma del estado. En un extremo del espectro (donde se encuentran, y cabe enfatizarlo, tanto las organizaciones supranacionales como las ONG locales) se asume que el papel del estado debería quedar sumamente restringido a una pocas actividades en las cuales los mercados no pueden optimizar las ganancias sociales $^{15}$. En el otro extremo, una amplia gama de actores -desde el "Consenso de Buenos Aires" hasta las OSN- están persuadidos de que el estado aún tiene un rol fundamental en la regulación de mercados estratégicos (Bradford, 1994; Castañeda y Mangabeira Unger, 1998; Stiglitz 1998a , 1998b, 1998c).

El debate acerca del nivel apropiado de regulación del estado involucra a áreas de política más específicas. Por ejemplo, para casi todos los fundamentalistas del mercado, la acción efectiva del estado requiere un sistema eficaz de tributación, pero todavía hay desacuerdo respecto de los medios y los niveles apropiados de dicha tributación; aunque hay un acuerdo creciente en lo referente a la efectividad de los impuestos al consumo, hay grandes discrepancias en cuanto a mantener o elevar los impuestos sobre el ingreso corporativo e individual. De igual modo, a raíz de las recientes crisis financieras recientes de Asia, Rusia y Brasil, muchos reformistas latinoamericanos de centro izquierda aceptan la inversión extranjera directa (particularmente en nuevas industrias pero no necesariamente en la privatización de empresas públicas), pero reclaman mecanismos selectivos (tales como el impuesto Tobin sobre las transacciones financieras, así como controles moderados sobre la cuenta de capitales, al estilo chileno) para atenuar la volatilidad de los mercados financieros internacionales y reducir la dependencia regional de los caprichos de los flujos de capital de cartera. También se ha generado un debate importante con respecto a cuánto deben intervenir los estados nacionales en la redistribución de activos -por ejemplo, entre las empresas manufactureras más competitivas y menos competitivas, entre grandes terratenientes y pequeños productores rurales, o de manera más general, entre los ricos y los pobres.

En segundo lugar, hay divergencias considerables respecto de la dirección deseada de la reforma laboral. Para algunos (ej. BID 1996 y 1998), el crecimiento económico sostenido y la creación de oportunidades de empleo requieren una flexibilización substancial de los mercados de trabajo, los cuales son una pieza central de los programas de liberalización ${ }^{16}$. Según otros, como la Organización Internacional del Trabajo (OIT) y los sindicatos obreros, ya ha sido implementada una flexibilización de facto sustancial, sin que esas reformas hayan logrado expandir significativamente las oportunidades de empleo. Desde este punto de vista, las reformas futuras deben ser más activistas y estar dirigidas directamente a la creación de empleo, a elevar las destrezas de trabajo y a incorporar las tecnologías apropiadas (CEPAL, 1997; Tokman, 1997; OIT, 1999). Está claro que este tema tiene una importancia crucial, dado que estas políticas afectan la postura de los sindicatos y los partidos políticos hacia las reformas de "segunda generación".

El tercer punto de disputa se refiere a la relación entre democracia, crecimiento económico y movilización política. Aunque existe un amplio consenso según el cual los estados tienen que ejercer un rol regulador importante contra la corrupción y el rent-seeking para asegurar la competencia y la transparencia del mercado, algunos sostienen que la participación social es imprescindible para lograr

adecuados para la regulación social y estatal. En el mismo trabajo, discutimos en más detalle las teorías de crecimiento endógeno.

14. Como lo señala Bulmer-Thomas (1996:312), “...lo que hace falta es un consenso entre los partidos políticos más importantes sobre la esencia de las reformas requeridas para mejorar la equidad en el largo plazo. El consenso sobre los elementos principales del [nuevo modelo económico] ya existe en la mayoría de los países de América Latina; es tiempo de extenderlo a los determinantes clave de la distribución del ingreso y la pobreza...".

15. Así "en una convergencia inesperada, el antiestatismo del neoliberalismo encontró un socio en la hostilidad basista hacia la burocracia, que cultiva el movimiento de ONG” (LEHMANN y BEBBINGTON, 1998:261).

16. El Banco Mundial desarrolla una línea dura con respecto a las reformas laborales: "...Aunque los reveses sufridos por intentos de liberalización del pasado han estado en parte influenciados por inadecuadas políticas del mercado laboral, no conocemos ningún intento reciente de liberalización en el que los problemas de desempleo hayan conducido a reveses..." (Primo BragA, NoguÉs Y RAJAPATIRANA, 1997:106). 
esos objetivos, mientras que otros son más cautelosos respecto de la incertidumbre potencial inherente a esa participación ${ }^{17}$. En verdad, para muchos partidos políticos, movimientos sociales y ONGs, la movilización política agresiva y el fortalecimiento de la capacidad de acción colectiva de los grupos subalternos o excluidos es fundamental para promover una mayor democratización ${ }^{18}$. Desde esta perspectiva, la movilización debería incorporar, en especial, a aquellos que han sido excluidos del poder (las mujeres, las minorías étnicas y raciales, los niños, los trabajadores del sector no organizado e informal) ${ }^{19}$. Tal como surge del "Consenso de Buenos Aires" mientras

\begin{abstract}
“... el mercado debería ser el principal distribuidor de recursos, [...] le corresponde al estado crear las condiciones para que las necesidades de los pobres se transformen en demandas efectivas que puedan ser procesadas" por el mercado (Castañeda, et al. 1998).
\end{abstract}

Es posible que una estrategia de movilización de este tipo provoque la oposición de un conjunto de actores sociales y políticos temerosos de cualquier cambio en el equilibro de poder prevaleciente. Por ejemplo, puede haber oposición de los policy-makers, las elites económicas y las organizaciones empresariales, quienes sostendrían que la movilización política genera inestabilidad política y por lo tanto podría hacer peligrar el equilibrio macroeconómico y el acceso a los flujos internacionales de capital. Algunos líderes de los partidos políticos también podrían oponerse a los esfuerzos para cambiar la agenda política y abrir el acceso a la toma de decisión a sectores más amplios de la población. También podría haber una fuerte oposición entre los sectores del movimiento laboral que vean amenazados sus intereses por algunas dimensiones de las reformas en cuestión. Finalmente, a pesar de que a menudo los pobres constituyen una clientela muy débil y desorganizada, los sectores sociales privilegiados y la clase media frecuentemente se articulan en "coaliciones de veto" oponiéndose a políticas que favorezcan a los primeros ${ }^{20}$. En el futuro, se puede esperar que diversos actores y fuerzas se aglutinen en diferentes combinaciones para oponerse al surgimiento de las coaliciones policéntricas.

En síntesis, los dos ejes que hemos identificado como implícitos en las discusiones sobre la tercera vía involucran distintos actores y modalidades/estrategias de acción. Los actores de cada eje convergen en algunas áreas de acción y reforma institucional, pero divergen en otras. En las dos secciones que siguen, discutimos en mayor detalle cada uno de estos ejes, y sus posibles despliegues en el futuro inmediato.

\section{EL PROBABLE DESARROLLO DESIGUAL DEL NUEVO RÉGIMEN INTERNACIONAL DE POLÍTICA}

¿Cuáles son las críticas a la estrategia de una tercera vía? Mas allá de la crítica a la alta vaguedad con que se ha formulado tal estrategia, vaguedad que llevaría a un alto grado de oportunismo político, el punto de partida de la crítica se ubica en la reformulación de la relación entre regulación estatal y regulación de mercados. En este sentido, se plantea la creación de oportunidades potenciales para la ciudadanía versus una redistribución de costos y beneficios cuyo efecto final sería aumentar la vulnerabilidad de la mayoría de las poblaciones afectadas por los cambios, argumentando que los diferentes intentos de reinventar el estado muy a menudo esconden una retirada de los mecanismos de protección social. Con el pretexto de buscar una mayor eficiencia administrativa o una descentralización de los servicios ofrecidos por el estado, las elites políticas buscarían, en efecto, recortar el gasto social. La tercera vía, así, representaría básicamente la nueva máscara del neoliberalismo en momentos en que los costos de la globalización y la apertura de mercados requerirían una mayor mediación política de las reformas en curso.

En Latinoamérica, esta postura crítica desconfía a su vez de la reformulación del Consenso de Washington y la “segunda generación” de reformas, considerándolas un esfuerzo de maquillar el mismo proyecto neoliberal, ahora con una capa superficial de políticas diseñadas para amortiguar (pero no evitar) sus impactos negativos para los sectores más vulnerables. Así, la apelación a una mayor participación de la sociedad civil escondería, en efecto, una creciente desmovilización de ésta frente a la ofensiva de los mercados, y el auge del individualismo posesivo y el consumismo desenfrenado.

Finalmente, la llamada tercera vía contribuiría directamente a la desmovilización y desarticulación de ciertas fuerzas progresistas clave. En efecto, en el contexto de crisis de la industrialización pesada de la era fordista, la tercera vía sería un mecanismo a través de cual se limitaría o socavaría

17. La posición anterior caracteriza el enfoque del Banco Mundial y del Banco Interamericano de Desarrollo. Véase también Williamson (1993) que defiende el fortalecimiento de los entes recaudadores de impuestos y una mavor autonomía de los bancos centrales para "aislarlos" de la política electoral, una idea que para los críticos tiene implicaciones potencialmente antidemocráticas. Para algunos representantes de la izquierda, como los socialistas chilenos y quizás para el FREPASO o el PRD, es esencial poner el acento en la rendición de cuentas a través de la participación pública. El confiar únicamente en la supervisión técnica de organismos regulatorios hace que se corra el riesgo de "captura" por los nuevos intereses monopólicos privados a ser regulados.

18. Por ejemplo, MARKOFF (1997) subraya que “...el gran reto del siglo veintiuno, si la democratización ha de tener un futuro significativo, será desarrollar formas de hacer que las estructuras de poder transnacionales, desde la 'Europa emergente' hasta el reto más difícil de las redes planetarias de las finanzas, respondan ante los que se vean afectados por sus acciones..."

19. Después de todo, como lo señala el "Consenso de Buenos Aires" “...en nuestras sociedades fragmentadas y heterogéneas, casi nadie es simplemente un ciudadano: la cantidad de grupos, sectores, minorías, regiones y grupos étnicos víctimas de alguna forma adicional de discriminación y opresión, que va más allá de las que ya están presentes en todas las sociedades, [genera] una política en constante efervescencia. Las mujeres, los jóvenes, los trabajadores, las comunidades indígenas, los negros y mulatos, las minorías con orientaciones sexuales específicas, los grupos étnicos oprimidos, las sectas religiosas marginalizadas y otros muchos grupos privados de la plenitud de sus derechos y aspiraciones, deben ser estimulados a movilizarse para conquistar [su] espacio y [perseguir] sus derechos e intereses..." (CASTAÑEDA, et al. 1998).

20. También es muy posible que muchos sindicatos limiten su acción política a apoyar a los trabajadores del sector formal (en vez de extender su apoyo a los desempleados o a los del sector informal), complicando así su participación como actores centrales en una coalición amplia. Ver O'DONNELL (1998). 
la fuerza política de actores tales como los sindicatos o la clase obrera. Asimismo, con el pretexto de evitar el efecto pernicioso de sistemas de seguridad para la ética de trabajo de los sectores más vulnerables de la ciudadanía, tal vía simplemente dejaría a estos sectores expuestos a la disciplina impuesta por los mercados globalizados, aumentando así la magnitud e impacto de la pobreza y la desigualdad.

Sintetizando, la postura crítica presenta a la tercera vía como un retroceso de las fuerzas progresistas y, en consecuencia, particularmente en Latinoamérica, una mayor erosión de la democracia, con una continuación del crecimiento económico desigual y una profundización de la desigualdad.

¿Cuál es la relevancia entonces de la tercera vía en el marco de las transformaciones políticas que están caracterizando a Latinoamérica? Volviendo a los paralelos históricos con el estado de bienestar, nuestro argumento es que se puede esperar un alto grado de diversidad en el predominio relativo de las reformas tipo tercera vía en la región. Esta diversidad, en el contexto de una reestructuración orientada hacia el mercado y la globalización acelerada tendrá que ver, por lo menos en parte, con la inédita, y hasta ahora poco probable, convergencia entre las más recientes versiones del discurso pro-equidad de los actores transnacionales y el discurso aggiornado de la izquierda democrática latinoamericana.

$\mathrm{Al}$ discutir las implicaciones de estas limitaciones para las potenciales coaliciones policéntricas, es útil considerar tres escenarios a modo de caminos alternativos hacia la globalización: el "camino inferior," el "camino medio" y el "camino superior" (Korzeniewicz y Smith, 2000). En el camino inferior, las elites políticas persiguen alianzas con algunas minorías estratégicas y buscan neutralizar la capacidad de muchas fuerzas sociales y políticas de comprometerse en una acción colectiva de oposición. En este escenario, la profundización del neoliberalismo y la estabilidad económica y política se ven facilitadas por una lógica dual del poder del estado (apoyo para la organización de la minoría aliada y desarticulación del resto), y por la distribución desigual de los recursos para apoyar prácticas clientelistas de corte neopopulista (Acuña y Smith, 1996; Roberts, 1995; Weyland, 1996). Este escenario de camino inferior a menudo se ve marcado por una falta de transparencia, un deterioro de los mecanismos de accountability y una corrupción extendida entre los funcionarios. Lógicamente, estas características de debilidad institucional y jurídica constituyen grandes obstáculos para el crecimiento económico sostenido. El escenario del camino inferior coloca todo el peso de los costos de transición de las reformas de mercado y de apertura de la economía a la competencia global en los pobres y en los sectores desorganizados de la sociedad. La pobreza creciente y la desigualdad se convierten en rasgos centrales de esta vía. La participación de los movimientos sociales contestatarios, las ONG y los partidos políticos reformistas en este escenario se ve por lo general limitada a su inclusión en redes clientelistas. El camino inferior, por lo tanto, no merece la calificación de tercera vía.

Es importante subrayar que el camino inferior no excluye el ejercicio de la política electoral. De hecho, en los ochen- ta y noventa muchos gobiernos de la región que han seguido este camino (por ejemplo, Argentina, Brasil, Perú) han llegado al poder a través de elecciones. En muchas instancias, el advenimiento de estos gobiernos por la vía electoral coincide con la hiperinflación y el fracaso catastrófico de las políticas heterodoxas de estabilización de fines de los ochenta (Smith, 1989). A pesar de las diferencias entre países y los esfuerzos periódicos por cambiar de camino y mejorar la calidad del crecimiento, ésta es la trayectoria que ha caracterizado a la mayoría de los países latinoamericanos desde la década de 1980.

El segundo escenario, el camino medio, corresponde a una tercera vía "conservadora" que combina: (1) una variante de reformas de mercado a ultranza y crecimiento económico sostenido; (2) un régimen democrático estable, relativamente consolidado, pero con características significativamente elitistas y excluyentes; y (3) una reducción consistente del desempleo y de la pobreza (lograda mediante la combinación de crecimiento, mayor inclusión y políticas sociales compensatorias), pero poco resultado en la reversión de las desigualdades persistentes en la distribución del ingreso y la riqueza. Simultáneamente, se adoptan políticas para mejorar la accountability y la transparencia, así como para atacar la corrupción y el clientelismo. Los partidos políticos de oposición tienen el rol de garantizar la gobernabilidad y los mecanismos neocorporativistas de intermediación incorporando a los actores sociales y económicos clave de modo de complementar al parlamento en el manejo de los conflictos sociales y en la negociación de políticas macro y sectoriales. Aunque periféricos con respecto a los partidos y los sindicatos, los movimientos sociales y las ONG tienden a representar un papel más activo en el diseño de políticas dentro de este escenario, aunque utilizan canales que a veces coinciden más con una versión de lo que podría rotularse como organizaciones "neo-" o "para-," más que como organizaciones "no-gubernamentales" (Schild, 1998).

La experiencia de Chile en los noventa se acerca mucho a está estrategia de camino medio. De hecho, Chile a menudo ha sido destacado con cierta exageración como un modelo de "crecimiento con equidad" a ser imitado por otras naciones de la región. Una serie de características acercan a Chile al camino medio más que al superior. Su éxito en el crecimiento económico se debe en parte a las políticas autoritarias implementadas por el régimen de Pinochet, y en gran medida a enclaves o mecanismos institucionales autoritarios (ej. reglas electorales que permiten una sobre-representación de las minorías conservadoras; los senadores "designados" no elegidos en el Congreso Nacional; fuerte representación militar en un Consejo de Seguridad Nacional dotado de amplios poderes de fiscalización sobre las autoridades civiles, etc.) (Moulian, 1997). A pesar de ello, la presencia de los partidos políticos relativamente fuertes, concomitantemente con ciertos rasgos de participación neo-corporativista más o menos institucionalizada de las cúpulas de las principales asociaciones empresariales y los sindicatos de los trabajadores, ha facilitado la negociación e implementación de políticas capaces de reducir significativamente la pobreza (Weyland, 1997). Sin embargo, la desigualdad se mantiene muy por encima de los niveles previos a 1973, sin lograr 
cerrar las brechas sociales bajo la nueva democracia (Martínez y Díaz,1996; Weyland, 1998) ${ }^{21}$.

Finalmente, en contraste con los dos caminos anteriores, en el escenario del camino superior al crecimiento -o tercera vía más "progresista" - y la integración en los mercados mundiales, los presidentes electos y sus equipos económicos y políticos podrían impulsar una reformulación del proceso de toma de decisiones. Un desempeño económico sólido, una distribución más equitativa del ingreso y la riqueza, y el abandono de las posturas hobbesianas, serían acompañados por la profundización relativa de la democracia procedimental ${ }^{22}$. La accountability y la transparencia prevalecerían en el ejercicio del gobierno. El progresivo fortalecimiento de la institucionalidad democrática y el estado de derecho tendería a atenuar la corrupción, el clientelismo y la impunidad. A su vez, en este escenario, los movimientos sociales y las ONG (y las redes transnacionales de las que forman parte) tendrían un rol más central en la promoción de la participación autónoma de grupos subalternos y en la ampliación de los espacios de interlocución democrática esenciales para el debate público. Los partidos políticos y otros actores sociales serían fortalecidos e incorporados más plenamente al diseño e implementación de políticas.

Dado que este enfoque del camino superior concibe una participación más activa del estado en la puesta en práctica de políticas sociales y el fomento del crecimiento ${ }^{23}$, existen razones teóricas convincentes (véase Bresser Pereira, Maravall y Przeworski, 1994; Acuña y Smith, 1996) para creer que un proyecto socialdemócrata aggiornado podría generar un equilibrio macroeconómico estable, con un crecimiento dinámico similar al logrado con la opción media. Sin embargo, el objetivo también alcanzaría un mayor énfasis en la equidad y en una compensación más generosa (a través de redes de seguridad, inversiones sociales y la adaptación de sus capacidades) para los "perdedores" de la integración competitiva en la economía global. Las comparaciones históricas y las consecuencias probables de una participación social y política ampliada en los procesos de toma de decisiones, hacen que las oportunidades distributivas de este escenario socialdemócrata sean, sin lugar a dudas, mucho más avanzadas que las estrategias del camino medio y del camino inferior.

El desafío para los defensores del camino superior y la tercera vía más progresista es el de ofrecer un liderazgo efectivo en el marco de la construcción de las coaliciones policéntricas del tipo reform-mongering (Hirschman, 1971) de modo de responder a las cambiantes preocupaciones de la clase media, los trabajadores formales e informales, las mujeres, los ambientalistas y los pueblos indígenas. Un esfuerzo de este tipo canalizaría la participación de estos sectores de modo de desarticular o minimizar el riesgo de las coaliciones de veto que apelen a la nostalgia por el proteccionismo del estado y a la ilusión de que los subsidios y los privilegios asociados a los comportamientos de rent-seeking, típicos del estilo de desarrollo basado en la sustitución de importaciones, pueden ser recuperados fácilmente. Hay esperanzas de que el tercer gobierno de la Concertación, ahora bajo el liderazgo del socialista Ricardo Lagos, logre ir más allá de camino medio característico de Chile en la etapa post-autori- taria para impulsar una tercera vía más transformadora que efectivamente ponga un énfasis mayor en medidas de política social tendientes a mejorar la distribución y la equidad, promoviendo la participación de los más postergados y excluidos ${ }^{24}$.

Los movimientos sociales y las ONG relacionadas con redes transnacionales pueden tener un papel esencial en el éxito de estos esfuerzos. Sin embargo, en cada uno de los tres caminos alternativos, la alianza de las ONGs con las SNOs puede jugar roles muy diferentes. Los optimistas podrían sostener que las características organizacionales de los movimientos sociales locales y de las ONG aumentarán la posibilidad de que las coaliciones policéntricas logren promover la estrategia del camino superior y profundizar la democratización más allá de la esfera político-institucional. No obstante, también existe la posibilidad de desenlaces más problemáticos, en los cuales el crecimiento de este tipo de organizaciones proporcione legitimidad a programas tecnocráticos o a líderes neopopulistas de derecha (ej. Alberto Fujimori o Carlos Menem) para continuar con el desmantelamiento del estado benefactor e, incluso, desarrollar nuevos lazos corporativos entre el estado y clientelas políticas entre los pobres y excluidos.

Cabe aquí trazar algunos paralelos con la adopción de los modelos de crecimiento keynesianos en la posguerra (Esping-Anderson, 1990). La adopción de estos modelos

21. No obstante las limitaciones de la nueva democracia chilena, los éxitos relativos en el campo social se destacan no sólo en comparación con el agravamiento de las brechas sociales en los países que han seguido el camino inferior (como la Argentina bajo Menem, por ejemplo) sino también con respecto a la experiencia brasileña bajo el gobierno de Fernando Henrique Cardoso, quien se declara socialdemócrata y quien ha participado en las discusiones de la Internacional Socialista y en varias reuniones internacionales con los principales ideólogos de la tercera vía como Tony Blair y Bill Clinton. Con respecto al carácter socialdemócrata del partido de FHC, el Partido da Social Democracia Brasileira (PSDB), véanse POWER (1997 y 2000). Para un análisis que enfatiza tanto los obstáculos político-institucionales como los desafíos internacionales a un proyecto reformista de corte social democrático en Brasil bajo FHC, ver SMITH Y MESSARI (2000).

22. Véase ACUÑa Y SMITH (1996) para más detalles sobre esta posibilidad. WEYLAND (1996 y 1997) presenta una perspectiva teórica algo diferente pero convergente, que le da significativa importancia a la "capacidad abarcativa" de las organizaciones de la sociedad civil e instituciones del estado, para la generación de resultados equitativos bajo condiciones democráticas. Se pueden encontrar interesantes propuestas para profundizar la democracia y aumentar la equidad en el modelo chileno en HARDY (1996) y en la COMISIÓN ECONÓMICA PSCH (1998), propuestas discutidas por economistas del partido socialista para un posible tercer gobierno de Concertación. Nótese también las semejanzas entre estas propuestas chilenas y el "Consenso de Buenos Aires" (CASTAÑEDA, et al. 1998), plataforma comentada anteriormente.

23. Esta estrategia representa un intento de basar la estabilidad democrática sobre pactos sociales y políticos amplios que le aseguraría a los principales actores colectivos que sus intereses no se vieran seriamente socavados con el ajuste estructural y las fuerzas del mercado global. Este escenario concuerda con propuestas que reclaman "...la construcción de un nuevo estado de desarrollo [que] resida en una mezcla de desregulación y de nueva regulación. Requiere que el estado camine por un sendero angosto, dejando operar al mercado sin sofocarlo y, al mismo tiempo, representando el papel en la coordinación y supervisión que las firmas privadas por sí solas no asumirían..." (CAVAROZZI, 1992: 682).

24. El hecho de que haya logrado avanzar más hacia la plena consolidación de las reformas de mercado (véase STALLINGS y PERES, 2000) con tasas de ahorro doméstico y inversión bruta superior al 25 por ciento del PBI es indudablemente un factor decisivo que facilitaría la ambición de la Concertación bajo Lagos de llevar a Chile hacia una tercera vía de tipo más progresista en el campo social. 
no sólo fue desigual según los contextos (el estado benefactor tuvo su mayor desarrollo en los países avanzados), sino que, además, estuvo acompañada por la democratización de algunos aspectos de la vida política y social (por ejemplo, en lo referente a las relaciones laborales) en algunos países, y por la dictadura y un corporativismo excluyente en otros. El desarrollo futuro del camino superior o tercera vía progresista de crecimiento con equidad puede caracterizarse por diferencias similares entre los países ${ }^{25}$

En efecto, la adopción de un modelo de crecimiento con equidad en América Latina puede verse obstaculizada por el legado específico de fuerzas políticas y arreglos institucionales en los diferentes países ${ }^{26}$. Pero lo que puede dar un ímpetu adicional a la estrategia de camino superior es precisamente el hecho de que, en definitiva, este tipo de estrategia sería sostenida por una amplia configuración de actores y fuerzas sociales. En primer lugar, el apoyo al modelo proviene de actores colectivos que operan por encima y por debajo de los arreglos estado-céntricos, y que van empujando gradualmente hacia una nueva matriz institucional determinada por nuevas formas organizacionales y alianzas políticas características de las coaliciones policéntricas. En segundo lugar, como el desempeño económico comparado es central, la adopción de la estrategia del camino superior puede facilitarse en la medida en que sea implementada en toda la región (e incluso fuera de la misma). Si la adopción de las estrategias del camino superior fuera acompañada por un éxito percibido en por lo menos un país, (mejorando los indicadores de crecimiento económico, el desempeño en los niveles de pobreza y desigualdad, y la profundización de la democracia), el modelo de crecimiento con equidad podría generar una "masa crítica" que contrarreste las percepciones negativas acerca de los costos de adoptar estrategias similares en otros países ${ }^{27}$.

En definitiva, el éxito relativo de cada uno de estos tres caminos esbozados puede medirse por su efectividad para superar el legado persistente de pobreza, desigualdad y autoritarismo en la región. El camino superior o tercera vía más progresista delineado de modo muy esquemático en este artículo, es menos una receta formal y más un esfuerzo preliminar para especificar algunas de las medidas y transformaciones congruentes con las metas igualmente esenciales de equidad y democracia. El logro de tal camino superior como vía más reformista hacia la globalización es una tarea difícil. El realismo y las experiencias históricas análogas advierten que es probable que poderosas fuerzas conservadoras intenten bloquear este tipo de experimento, y en muchos países es posible que esas fuerzas logren, en diferentes grados, imponer sus preferencias contra un renovado modelo socialdemócrata. Por consiguiente, sin duda es posible, y quizás hasta probable que el resultado sea estable pero lejos de promover efectivamente una profundización democrática y crecimiento con equidad ${ }^{28}$.

En síntesis, la experiencia histórica con previos RIPs sugiere que el desarrollo futuro de la tercera vía en el mundo, y particularmente en América Latina, será altamente desigual. En esta región, tal vía podría caracterizarse por el predominio de caminos inferiores y medios hacia la democracia. Pero en última medida, tales escenarios dependen no sólo de la articulación interna de fuerzas progresistas dentro de cada país, sino también de la medida en que el RIP socialdemócrata converja con el desarrollo efectivo de la tercera vía como SGB.

\section{LOS OBSTÁCULOS AL SISTEMA GLOBAL DE BIENESTAR}

Mas allá de las probables irregularidades en el desarrollo institucional de la tercera vía como régimen internacional de política, podemos mencionar tres obstáculos fundamentales para el futuro de tal vía como sistema global de bienestar.

Primero, la expansión de los mercados es limitada en ciertas áreas clave. Como nos recuerda Albert Hirschman (1970), la ciudadanía no limita sus esferas de acción al ejercicio de su "voz". La "salida", es decir, la participación en mercados, también constituye un mecanismo importante de acción. Pero en este sentido resulta paradójico que los mercados laborales se mantengan tan regulados como en la mayoría del siglo XX: los estados nacionales, particularmente aquellos de más altos ingresos, mantienen el privilegio de regular la entrada y salida de sus fronteras, y han generado últimamente diversas formas de movimientos políticos (frecuentemente, pero no siempre, de tono conservador) que buscan fortalecer aún más la protección frente a la migración internacional. Así, los mecanismos de mercado que

25. Véase KORZENIEWICZ Y SMITH (2000) para un esfuerzo inicial de avanzar en el análisis de este tema.

26. Este es un terreno muy inexplorado sobre el que podrían tejerse hipótesis contradictorias. Sería necesario una cuidadosa investigación com parativa para identificar las diferentes constelaciones de factores (tradiciones de clientelismo y exclusivismos, cohesión de los aparatos del estado, fuerza y "capacidad de abarcar" de las organizaciones de clases y sectoriales, etc.) que favorecen o bloquean la aparición y el éxito de las coaliciones policéntricas de tinte socialdemócrata.

27. Además, como lo sugiere la crisis financiera actual y los efectos de contagio en la región, la globalización choca con una amplia gama de gru pos internos, incluyendo los empleadores y la clase media. Por consiguiente, es posible que esos grupos encuentren cada vez más atractivo el abandonar el camino inferior vinculado a las estrategias maximalistas con resultados distributivos nulos, cuando observen que la matriz de retribución se vuelve muy negativa, es decir, cuando los riesgos de mantenerse separado superen a los riesgos de la cooperación. Los incipientes debates sobre los beneficios de los controles de los flujos de capital, los regímenes de las tasas de cambio, etc. y la ventaja de las políticas industriales, entre economistas y elites de negocios menos globalizadas, sindicatos, consumidores, que de pron to se han asustado con la globalización financiera, pueden ser indicativos del hecho de que las perspectivas de las coaliciones policéntricas y de las alternativas del camino superior para la socialdemocracia pos-Fordista, después de todo, puedan no ser tan "utópicas".

28. Cabe observar también que cada una de las vías reseñadas puede generar ciertas dinámicas que aseguren su sustentabilidad política y social aún en un contexto de creciente desigualdad entre hogares. Por ejemplo, si es acompañada por crecimiento económico, la mayor desigualdad generada por la globalización puede conllevar un mayor bienestar y alivio de la pobreza, y/o una percepción generalizada que las tendencias polarizantes son compensadas por una alta movilidad social. Asimismo, aún sin un vigoroso crecimiento económico, y en un contexto de mayor desigualdad entre hogares, podrían reducirse otras dimensiones de desigualdad (por ejemplo entre hombres y mujeres, entre sectores urbanos y rurales, entre indígenas y no-indígenas), generando un apoyo selectivo pero crucial a la expansión de los mercados. Finalmente, la sustentabilidad de una estrategia no depende sólo de su impacto social, sino de la capacidad de grupos opositores de presentar propuestas que sean consideradas como alternativas y viables. 
a fines del siglo XIX y comienzos del XX atenuaron las disparidades de ingreso a nivel global, continúan a comienzos del siglo XXI en su mayor parte restringidos. La restricción de estos mercados es parte esencial de la reproducción de desigualdades a nivel global, y las versiones contemporáneas de la tercera vía, particularmente en países de altos ingresos, hasta ahora no se han mostrado dispuestos a confrontar este problema.

Segundo, considerando los paralelos históricos, es claro que aunque el auge del estado de bienestar llevó a una cierta convergencia entre países pobres y ricos desde el punto de vista de los niveles relativos de gasto fiscal y social (ver Figura 1), los arreglos implícitos en el estado de bienestar tuvieron un desarrollo altamente desigual. Por ejemplo, en el período de posguerra tendió a prevalecer la democracia en los países de alto desarrollo económico, mientras que los países de desarrollo medio o bajo se caracterizaron por la prevalecencia de dictaduras y autoritarismo (Korzeniewicz y Awbrey, 1992).

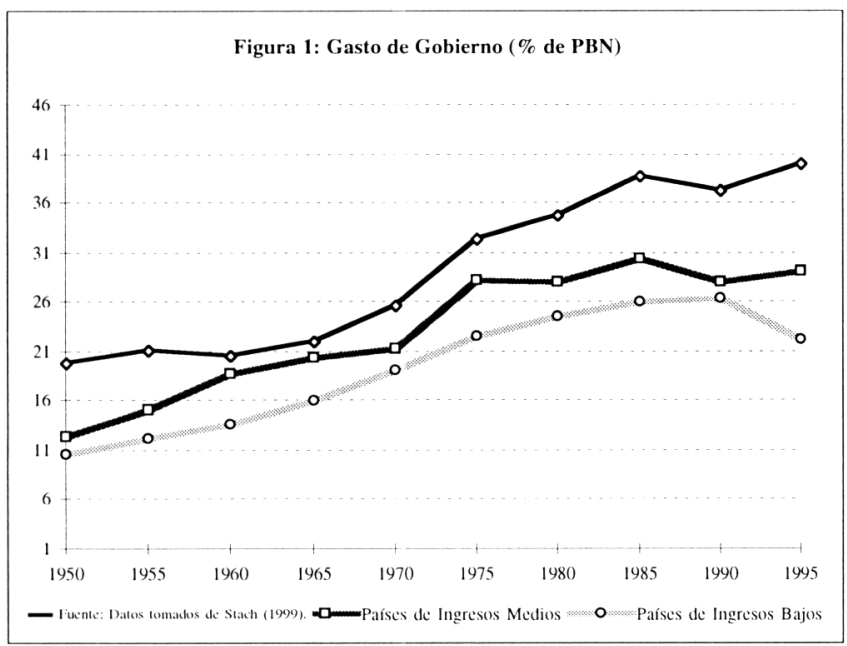

Por otra parte, los niveles absolutos de gasto fiscal (en general) y social (en particular), debido a la pronunciada desigualdad en nivel de desarrollo económico, se reflejan también en una profunda desigualdad a través del mundo. Como lo indica la Figura 2, los niveles de gasto social (educación, salud) son mucho más elevados en los países de altos ingresos.

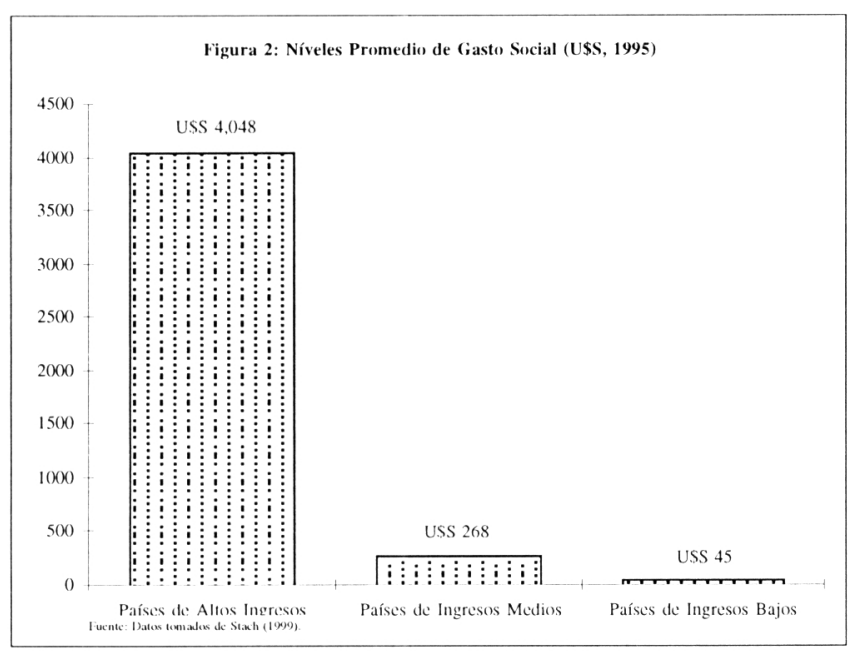

Las nuevas teorías de crecimiento endógeno, muy de boga entre las instituciones supranacionales que buscan justificar la necesidad de una "segunda generación" de reformas, nos sugieren que la inversión social es un elemento clave del crecimiento económico y, por ende, de las ventajas comparativas en los mercados internacionales ${ }^{29}$. Los datos presentados arriba sugieren que nos encontramos ante un círculo vicioso, donde la inversión social es limitada en países pobres por bajos niveles de producción de riquezas, pero donde los bajos niveles de inversión social aseguran la inevitabilidad de aquellos bajos niveles de producción de riquezas $^{30}$. Así como en el caso de los mecanismos de mercado, los límites de la tercera vía y las posibilidades reales de los futuros arreglos institucionales tendrán mucho que ver con su capacidad de ofrecer mecanismos efectivos para manejar esta problemática.

Existen dos mecanismos clave que servirían para confrontar directamente estos patrones de desigualdad. A nivel de mercado, mediante la eliminación de restricciones nacionales a los flujos migratorios y las exportaciones de países de bajos y medianos ingresos. A nivel de regulación, mediante un traslado de recursos de países de altos ingresos hacia países de bajos ingresos, con el propósito de aumentar substancialmente la inversión social dentro de estos últimos. Políticamente, los dos tipos de mecanismos son de muy difícil construcción, y hasta ahora, con muy pocas excepciones, los partidarios de la tercera vía, particularmente en los países de altos ingresos, no se han mostrado dispuestos a desarrollar una agenda explícita en torno a estos temas $^{31}$. Las razones son claras: si la implementación de la tercera vía a escala nacional dentro de los países de altos ingresos encuentra los obstáculos organizativos (especialmente la ausencia de bases sociales) que mencionaba Reich, estos obstáculos se multiplican exponencialmente cuando se propone construir nuevos arreglos institucionales a nivel global, arreglos que podrían amenazar los intereses de bloques políticos importantes. Un "New Deal" global en este sentido representaría un ejercicio de imaginación e implementación política sin precedentes.

El tercer punto tiene que ver con la dinámica de la convergencia señalada entre organizaciones supranacionales y las organizaciones no-gubernamentales. Esta dinámica supone la creciente adopción de mecanismos democráticos de representación y decisión. Esto, a su vez, apunta a dos dimensiones: la relación entre organizaciones supranacionales y las ONGs, por un lado, y la dinámica interna de las propias ONGs, por otro.

En torno a la relación entre organizaciones supranacionales y ONGs, una perspectiva pesimista enfatizaría que las primeras son organizaciones que representan principalmente los intereses de los gobiernos de los principales países

29. Así, entre otros, Jeffrey Sachs ha enfatizado recientemente que la falta de inversiones en educación y tecnología en países pobres contribuye a un círculo vicioso, donde esta falta de inversiones evita la participación en el tipo de innovaciones que asegura el acceso a mayor riqueza.

30. En gran medida la crisis del estado de bienestar tiene mucho que ver con esta dinámica.

31. Para un provocativo análisis de esta problemática, ver FAGEN (1978). 
contribuyentes que financian programas de tales organizaciones. Desde tal punto de vista, las organizaciones supranacionales, como el Banco Mundial o el BID, representarían las vanguardias organizativas de las elites que se beneficiarían con las reformas impulsadas por el neoliberalismo y la globalización. Aquí la participación de ONGs y movimientos sociales aliados se limitaría a una superficial interacción orientada a legitimar los programas desarrollados "desde arriba" por las organizaciones supranacionales.

Esta dinámica internacional, a su vez, condiciona fuertemente la dinámica interna de las ONGs, las cuales se verían afectadas por un proceso incipiente de burocratización, y por un liderazgo frecuentemente seducido ya sea por las posibilidades de empleo en el estado u organizaciones supranacionales, o por altos salarios que representarían una explotación de los sectores sociales que las ONGs buscarían representar.

En este contexto, las diversas propuestas de tercera vía, basadas en la necesidad de mejorar el acceso de sectores excluidos a la educación y a la salud, asegurando así el acceso de los pobres a las oportunidades abiertas por el mercado, abre involuntariamente un espacio de debate político alrededor de las oportunidades y/o los límites de las propuestas existentes, para así generar un debate sobre qué medidas adoptar para avanzar el futuro de la tercera vía como SGB

\section{ESTRATEGIAS ALTERNATIVAS DESDE "ARRIBA", “ABAJO" Y "AFUERA"}

Considerando los paralelos históricos mencionados y teniendo en cuenta las posibles irregularidades y obstáculos en el desarrollo de la tercera vía tanto a nivel nacional como a nivel global, surgen dos nuevas preguntas: ¿Cuál es la forma más adecuada de participar en la definición de los nuevos arreglos institucionales? Y ¿ cuáles son los objetivos más meritorios? Muchas veces nos atrae la posibilidad de identịficar un único camino de acción posible, una vía correcta para el futuro. Aquí, en cambio, evitaremos tal simplificación, para indicar que diversos actores elegirían diferentes caminos de acción, y que cada uno de estos caminos podría contribuir, a veces inesperadamente, a diversas construcciones y de nuevos arreglos institucionales.

Así, para algunos, el marco más efectivo de acción involucra la participación directa en la construcción de un SGB, ya sea a través de la reforma social de organizaciones supranacionales, o a través del trabajo de ONGs y los movimientos sociales. Para otros, la efectividad de la reforma social pasa por la articulación de fuerzas políticas en torno a proyectos nacionales de reforma, ya sea coordinando la formulación de programas adecuados desde las cumbres de la negociación política y/o la administración pública, o promoviendo la autonomía y postura crítica de las fuerzas sociales y políticas organizadas desde abajo. Finalmente, también debe considerarse la posibilidad de que, encarnando una lógica más profundamente antisistémica, los marginados de los arreglos institucionales de cada eje decidan "patear el tablero" y romper con las reglas de juego.
Históricamente cada una de estas estrategias -ya sea desde distintos ejes, o desde "arriba", "abajo" y "afuera" - fue de gran importancia en la definición del estado de bienestar. Los agentes políticos y administrativos fueron de gran importancia en la definición de proyectos de gobierno que produjeron mayor o menor crecimiento económico, mayor o menor marginación social, mayor o menos transparencia y mayor o menor democracia. Recordemos que, frecuentemente, la participación directa o indirecta de representantes de base (como líderes sindicales) en estos proyectos fue leída como traición a los intereses estratégicos de los sectores representados. Así, muchos le dieron gran valor a la autonomía frente a los proyectos de gobierno -autonomía a menudo practicada por partidos políticos, sindicatos obreros, organizaciones de campesinos-, etc. Sin embargo, muy a menudo esta autonomía en última instancia también sirvió para legitimar la propia existencia de un estado de bienestar que lograra generar mayor integración social y política. En muchos casos, la búsqueda de autonomía (a veces forzada por estados autoritarios o semi-autoritarios) llevó a romper con las reglas de juego planteando reglas alternativas (como en el comunismo) y en otros casos contribuyendo a un empate político marcado por crisis y violencia. Pero aún en estos contextos de rechazo a las reglas del juego, la constitución de modelos alternativos de desarrollo fue de gran importancia en la definición de la agenda del estado de bienestar.

Cabe esperar una dinámica similar en los futuros caminos de acción. Para algunos, los cambios institucionales o administrativos desplegados desde arriba son promisorios ya sea desde el punto de vista de objetivos éticos (por ejemplo, integración social, crecimiento económico, o profundización de la democracia) y beneficios personales, o un equilibrio óptimo entre los dos. En este marco, podemos esperar una creciente integración entre las esferas de organizaciones supranacionales, agencias gubernamentales y algunas ONGs. Otros, en cambio, podrían perseguir una autonomía crítica frente a estos agentes políticos y administrativos. En este sentido, se espera una creciente coordinación de organizaciones de la sociedad civil, ya sea a nivel de alianzas políticas de oposición, o de redes o cadenas internacionales de coordinación (estas redes seguramente tendrán una creciente jerarquización y burocratización, aunque, como en el caso del movimiento obrero, no debemos subestimar la capacidad de tales mecanismos de generar movimientos de base que cuestionen su propio liderazgo). Finalmente, una tercera alternativa es la búsqueda de una estrategia de "patear el tablero", ya sea a través de modelos autárquicos de desarrollo nacional (como en la estrategia yugoslava o iraquí), movimientos de liberación (como el caso de los zapatistas en México) o la participación en nichos ilegales de producción y distribución de riquezas (como el caso del narcotráfico). El mundo de violencia implícito en estas alternativas promete reemplazar a la amenaza del comunismo como referente clave de las futuras estrategias de reforma social.

\section{CONCLUSIÓN}

Entre el optimismo y el pesimismo se ha delineado un terreno fructífero para la interpretación del futuro de la ter- 
cera vía en la región. La tercera vía es expresión constitutiva de dos ejes distintos de cambio. El primero representa el proyecto de la tercera vía como sistema global de bienestar. Este eje es expresión del "doble movimiento" ya mencionado: la expansión de mercados acompañada por nuevas lógicas de regulación y resistencia en lo social y lo político, llevando al surgimiento de inéditos arreglos institucionales en el ámbito global. El segundo eje representa el proyecto de la tercera vía como régimen internacional de política. La acción de actores organizadas desde "arriba" “abajo," y "afuera” del sistema global de bienestar y del régimen internacional de política, produce áreas de convergencia pero también de conflicto entre los dos ejes, condicionando el futuro de la democracia y la equidad.

En general, creemos que se puede esperar un desarrollo irregular de la tercera vía como régimen internacional de política tanto como sistema global de bienestar. En América Latina, esta irregularidad podría traducirse en límites o barreras para la efectividad de los mecanismos democráticos, la transparencia administrativa y la capacidad de generar crecimiento e inclusión social. Aún más, la tercera vía como régimen internacional de política también se ve jaqueada por la falta de voluntad política en los países de altos ingresos para transformar ciertas dinámicas cruciales que subyacen a la desigualdad del capitalismo como sistema global, como economía-mundo. Sin embargo, más allá de los problemas indicados, creemos que la discusión sobre los dos ejes de la tercera vía abre importantes espacios como desafío intelectual, en la línea de lo que Immanuel Wallerstein (1995a y 1995b) llama "utopística" (utopistics). Esta discusión es relevante porque, al perseguir como objetivo la desarticulación de los mecanismos de desigualdad, exige repensar los arreglos institucionales globales que han caracterizado al capitalismo a través de su historia.

\section{BIBLIOGRAFÍA}

Acuña, Carlos H., y William C. Smith (1996): "La economía política del ajuste estructural: La lógica de apoyo y oposición a las reformas neoliberales". Desarrollo Económico N. 141, Vol. 36 (abril-junio): 355-390.

Alvarez, Sonia E., Evelina Dagnino y Arturo Escobar (comps.) (1998): Cultures of Politics. Politics of Culture: Re-Visioning Latin American Social Movements. Boulder: Westview Press. Alvarez, Sonia E. (1998): "Latin American Feminisms 'Go Global': Trends of the 1990s and Challenges for the New Mellennium." Pp. 293-324 en S.E. Alvarez, E. Dagnino y A. Escobar (comps.): Cultures of Politics. Politics of Culture: Re-Visioning Latin American Social Movements. Boulder: Westview Press.

Banco Mundial (1998): Beyond the Washington Consensus: Institutions Matter. Washington, D.C.: The World Bank.

- (2000): "Wolfensohn Calls for Globalization With a Human Face". 16 de febrero.

Berger, Suzanne y Ronald Dore (comps.) (1996): National Diverity and Global Capitalism. Ithaca: Cornell University Press.

Borx, Carles (1998): Political Parties, Growth and Equality: Conservative and Social Democratic Economic Strategies in the World Economy. Cambridge: Cambridge University Press.

BID. (1998): Economic and Social Progress in Latin America, 1998 1999 Report: Facing Up to Inequality in Latin America. Washington, D.C.: Inter-American Development Bank.
- (2000): Economic and Social Progress in Latin America, 2000 Report: Development and Beyond. Washington, D.C.: InterAmerican Development Bank.

BRAdFord, Colin I. Jr.(1994): "Redefining the Role of the State: Political Processes, State Capacity and the New Agenda for Latin America." En C.I. BRADFORD, Jr. (comp.): Redefining the State in Latin America. Paris: Organization for Economic Cooperation and Development (OECD).

Bulmer-Thomas, Victor (1996): “Conclusions.” Pp. 295-314 en V. Bulmer-Thomas (comp.): The New Economic Model in Latin America and its Impact on Income Distribution and Poverty. New York: St. Martin's Press.

CASTAÑEDA, Jorge, et al. (1998): "Después del neoliberalismo: Un nuevo camino". El Nuevo Herald Digital. 11 de enero.

Cavarrozzi, Marcelo (1992): "Beyond Transitions to Democracy in Latin America". Journal of Latin American Studies 24 : 665 684.

Chalmers, Douglas A., Scott P. Martin y Kerianne Piester (1997): "Associative Networks: New Structures of Representation for the Popular Sectors?” Pp. 544-82 en D.A. Chalmers, C.M.

Vilas, K. Hite, S.B. Martin, K. Piester y M. Segarra (comps.): The New Politics of Inequality in Latin America: Rethinking Participation and Representation. New York: Oxford University Press.

Clinton, Bill. (1999): "Remarks By The President at University of Chicago Convocation Ceremonies". White House, Office of the Press Secretary. 12 junio.

COMISIÓn ECONÓMICA PSCH (Partido Socialista de Chile) (1998): "Hacia un desarrollo en equidad y ciudadanía para todos: Base de una Plataforma para el Tercer Gobierno de la Concertación Democrática 2000-2006”. Borrador para el Encuentro de los Economistas Socialistas. 29 de marzo.

Dagnino, Evelina (1998): "Culture, Citizenship, and Democracy: Changing Discourses and Practices of the Latin American Left”. Pp. 33-92 en S.E. Alvarez, E. Dagnino y A. Escobar (comps): Cultures of Politics. Politics of Culture: Re-Visioning Latin American Social Movements. Boulder: Westview Press.

Dahrendorf, Ralf (1999): "The Third Way and Liberty: An Authoritarian Streak in Europe's New Center". Foreign Affairs 78(5) (Septiembre-Octubre): 13-17

ESPING-ANDERSON, Gosta (1990): The Three Worlds of Welfare Capitalism. Princeton, NJ: Princeton University Press.

FAGEN, Richard R. (1978): "Equity in the South in the Context of North-South Relations". Pp. 163-214 en Albert Fishlow, Carlos F. Díaz-Alejandro, Richard R. Fagen y Roger D. HANSEN (comps.): Rich and Poor Nations in the World Economy. Nueva York: McGraw-Hill.

Financial Times (2000): "Latin America: Region No Better Off Now, says World Bank". 4 de febrero.

GarretT, Geoffrey (1998): Partisan Politics in the Global Economy. Cambridge: Cambridge University Press

GIDDENS, Anthony (1998): The Third Way: The Renewal of Social Democracy. Cambridge: Polity_Press and Blackwell Publishers, Ltd.

- (2000): The Third Way and its Critics. Cambridge: Polity Press y Blackwell Publishers, Ltd.

Halpern, David y David Mikosz (comps.) (1998): "The Third Way: Summany of the NEXUS On-Line Discussion". <www. netnexus.org/library/papers/32way.html>.

Hardy, Clarissa, et al. (1996): Reflexiones socialistas sobre Chile. Santiago: Ediciones La Liebre Ilustrada.

Held, David, Anthony MCGrew, David GoldblatT y Jonathan PerRaton (Comps.) (1999): Global Transformations:Politics, Economics and Culture. Stanford, Calif.: Stanford University Press. 
HiRsCHMAn, Albert O. (1970): Exit, Voice and Loyalty: Responses to Decline in Firms, Organizations, and States. Cambridge: Harvard University Press.

- (1971): A Bias for Hope: Essays on Development and Latin America. New Haven: Yale University Press.

JELIN, Elizabeth (1997): "Emergent Citizenship or Exclusion? Social Movements and Non-Governmental Organizations in the 1990s". Pp. 79-104 en William C. SMITH y Roberto Patricio KorzeniewiCz (comps.): Politics, Social Change, and Economic Restructuring in Latin America Boulder, Colo.: North-South Center/Lynne Rienner.

- (1998): "Toward a Culture of Participation and Citizenship: Challenges for a More Equitable World". Pp. 405-414, en Sonia E. Alvarez, Evelina Dagnino y Arturo EsCobar (comps.): Cultures of Politics, Politics of Cultures: Re-visioning Latin American Social Movements, Boulder, Colo.: Westview.

KeOHANE, Robert O.y Helen V. MiLner (comps.) (1996): Internationalization and Domestic Politics. Nueva York: Cambridge University Press.

Kitschelt, Herbert, et all. (1999): Continuity and Change in Contemporary Capitalism. Cambridge: Cambridge University Press.

KLEIN, Rudolf y Anne Marie RAFFERTy (1999): "Rorschach Politics: Tony Blair and the Third Way". The American Prospect 45 (julio-agosto) (versión on line).

KorZENIEwiCZ, Roberto Patricio y Kimberly AwBrey (1992): "Democratic Transitions and the Semiperiphery of the WorldEconomy", Sociological Forum 7(4): 609-640.

Korzeniewicz, Roberto Patricio y William C. SMITH (2000): "Poverty, Inequality and Inequality in Latin America: Searching for the High Road to Globalization", Latin American Research Review 35(3): 1-35.

Lehmann, David y Anthony BebBington (1998): "NGOs, the State, and the Devleopment Process: The Dilemmas of Institutionalization". Pp. 251-270 en Menno VellingA (ed.): The Changing Role of the State in Latin America. Boulder, Colo.: Westview Press.

Maravall, José María, Luiz Carlos Bresser Perreira y Adam PRZEWORSKI (1993): Economic Reforms in New Democracies: A Social-Democratic Approach. Cambridge: Cambridge University Press.

- (1994): "Economic Reforms in New Democracies: A SocialDemocratic Approach". Pp. 181-212 en William C. SMITH, Carlos A. Acuña y Eduardo A. Gamarra (comps.): Latin American Political Economy in the Age of Neoliberalism: Theoretical and Comparative Perspectives. Miami, Fla.: NorthSouth Center Press

MARKOFF, John (1997): "Really Existing Democracy: Learning from Latin America in the Late 1990s”, New Left Review 223 (May-June) : 48-68.

MARTínEZ, Javier y Alvaro DiAz (1996): Chile: The Great Transformation. Washington, D.C.: Brookings Institution y UNRISD.

MERKEL, Wolfgang (1999): "Las terceras vías de la socialdemocracia en el 2000”, El País, 20 julio.

Moulian, Tomás (1997): Chile actual: Antonomía de un mito. Santiago: Arcis Universidad/Ediciones LOM.

NELSON, Paul (1999): "Whose Civil Society? Whose Governance? Decision-Making and Practice in the 'New' Agenda at the Inter-American Development Bank and the World Bank". Buenos Aires: FLACSO/Argentina.

New York Times (1999): "Triumphant, The Left Asks What Else It Is”. 21 de noviembre.

- (1999): "Clinton, a Renaissance Guy, Paints the Globe Bright" 22 de noviembre.

OFFE, Claus (1984): Contradictions of the Welfare State. Baltimore, MD: MIT Press.
O’Donnell, Guillermo (1998): "Poverty and Inequality in Latin America. Some Political Reflections”. Pp. 49-74 en V. TOKMAN y G. O'Donnell (comps): Poverty and Inequality in Latin America. Issues and New Challenges. Notre Dame: University of Notre Dame Press.

OIT (Organización Internacional del Trabajo) (1999): Panorama laboral de América Latina y el Caribe, 1999. Lima: Oficina Regional de la OIT.

PAís (EL) (1999): "La Internacional Socialista reclama la supremacía de la política sobre el mercado". 9 noviembre.

- (1999): "Socialistas Globales". 10 de noviembre.

- (1999): "Los líderes socialistas cierran su congreso con un consenso ante los retos de la globalización”. 11 de noviembre.

- (1999): "Clinton defiende la 'tercera vía' para superar las diferencias en la familia socialdemócrata”. 22 de noviembre.

- (1999): "Los modelos del capitalismo: Políticos e intelectuales debaten en Madrid los problemas de la globalización”. 26 de noviembre

- (1999): "La 'tercera vía' tendrá que ser distinta en cada país" Entrevista a John Gray. 29 de noviembre.

- (1999): "El liberalismo 'de izquierda'” Sami Nair. 17 de diciem bre.

POLANYI, Karl (1947): La gran transformación: Los orígenes politicos y económicos de nuestro tiempo. Buenos Aires: Editorial Claridad.

POwER, Timothy J. (1997): "Quais São os Valores da Social De mocracia Hoje? Algumas Reflexões Comparadas sobre a Crise de Identidade do PSDB". Political Science Department, Louisiana State University. Mimeo.

- (2000): "Blairism Brazilian Style? The PSDB and the Cardoso Experiment in Reformist Social Democracy”. Trabajo presentado en el Congreso Mundial de la Asociación Internacional de Ciencia Política. Québec, Canada. 1-5 de Agosto.

PrEZWORSKI, Adam (1985): Capitalism and Social Democracy. Nueva York: Cambridge University Press.

- En prensa. "How Many Ways Can Be Third?" In Andrew GLYN (comp.): Economic Policy and Social Democracy, Oxford: Oxford University Press.

Primo Braga, Carlos A., Julio Nogués and Sarath Rajapatirana (1997): "Latin America and the Caribbean in the World Economy”. Pp. 93-119 in S.J. Burki, S. EdWARDS, and S.R. AIyer (comps.): Annual World Bank Conference on Development in Latin America and the Caribbean 1995: The Challenges of Reform. Washington, D.C.: The World Bank.

ReICH, Robert B (1997): Locked in the Cabinet. Nueva York: Knopf.

- (1999): "We Are All Third Wayers Now", American Prospect 43 (mayo-Abril): 46-51.

ROBERTS, Kenneth M. (1995): "Neoliberalism and the Transformation of Populism in Latin America: The Peruvian Case", World Politics 48 (1): 82-116.

RugGIE, John Gerard (1982): "International Regimes, Transactions, and Change: Embedded Liberalism in the Postwar Economic Order", International Organization 36 (2): 379-415.

SCHILD, Verónica (1998): "New Subjects of Rights? Women's Movements and the Construction of Citizenshipom the 'New Democracies'”. Pp. 93-117 en S.E. Alvarez, E. Dagnino y A. Escobar (comps.): Cultures of Politics. Politics of Culture: ReVisioning Latin American Social Movements. Boulder: Westview Press.

SFERZA, Serenella (1999): "What is Left of the Left? More Than One Would Think", Daedalus 128(2) (Primavera): 101-126.

SMITH, William C. (1989): "Heterodox Shocks and the Political Economy of Democratic Transition in Argentina and Brazil". Pp. 138-168 en William CANAK (comp.): Lost Promises: Debt, Austerity and Development in Latin America. Boulder: Westview Press. 
- (1998): "Democracia, equidad y globalización: Del Consenso de Washington hasta el Consenso del Sur... y más allá?”. Pp. 111-123 in F. Rotas Aravena (comp.): Globalización, América Latina y la Diplomacia de Cumbres. Santiago, Chile: Facultad Latinoamericana de Ciencias Sociales.

SMITH, William C. y Nizar Messari (2000): "Democracy and Reform in Cardoso's Brazil: Caught Between Clientelism and Global Markets?" En Jeffrey STARK (ed.): The Challenges of Change in Latin America' and the Caribbean, Boulder, Colo: North-South Center Press/Lynne Rienner.

STACH, Angela (1999): A World-Economic Analysis of State Growth in the Twentieth Century. M.A. Thesis, Department of Sociology, University of Maryland, College Park.

Stallings, Barbara y Wilson Peres (2000): Growth, Employment, and Equity: The Impact of the Economic Reforms in Latin America and the Caribbean. Washingtion, D.C.: The Brooking Institution Press y la Comisión Económica para América Latina y El Caribe.

StiglitZ, Joseph E. (1998a): "More Instruments and Broader Goals: Moving Toward the Post-Washington Consensus". 1998 WIDER Annual Lecture (Helsinki), <www.worldbank.org/ html/extdr/extme/js-010798/wider.htm>.

- (1998b): "Redefining the Role of the State". Discurso para el Décimo Aniversario del Instituto de Investigaciones del MITI (Tokio), <www.worldbank.org/html/extdr/extme/jssp031798. htm>.

- (1998c): "Responding to Economic Crises: Policy Alternatives for Equitable Recovery and Development". North-South Institute (Ottawa, Canada). September 29. <www.worldbank. org/html/extdr/extme/jssp092998.htm>.
Tokman,Victor E. (1997): "Jobs and Solidarity: Challenges for Post-Adjustment in Latin America”. Pp. 349-71 in L. EMMERI (ed.): Economic and Social Development Into the XXI Century. Washington, D.C.: Inter-American Development Bank.

Tussie, Diana (ed.) (2000): Luces y sombras de una nueva relación: El Banco Interamericano de Desarrollo, el Banco Mundial y la sociedad civil. Buenos Aires: FLACSO/Temas Grupo Editorial.

WALLERSTEIN, Immanuel (1995a): "The Collapse of Liberalism". Pp. 232-51 in I. Wallerstein. After Liberalism. Nueva York: The New Press.

- (1995b): "The Agonies of Liberalism: What Hope Progress?" Pp. 252-71 in I. Wallerstein. After Liberalism. Nueva York: The New Press.

Weyland, Kurt (1996): Democracy Without Equity: Failures of Reform in Brazil. Pittsburg: University of Pittsburgh Press.

- (1997): "Growth with Equity in Chile's New Democracy?" Latin American Research Review 32(1): 37-67.

- (1998): "Economic Policy in Chile's New Democracy". Journal of Interamerican Studies and World Affairs 41(3): 67-96.

Williamson, John (1990): The Progress of Policy Reform in Latin America. Washington, D.C.: Institute of International Economics.

- (1993): "Democracy and the "Washington Consensus". World Development 21(8): 1329-36.

- (1997): “The Washington Consensus Revisited”. Pp. 48-61 in L. EMMERI (ed.): Economic and Social Development Into the XXI Century. Washington, D.C.: Inter-American Development Bank.

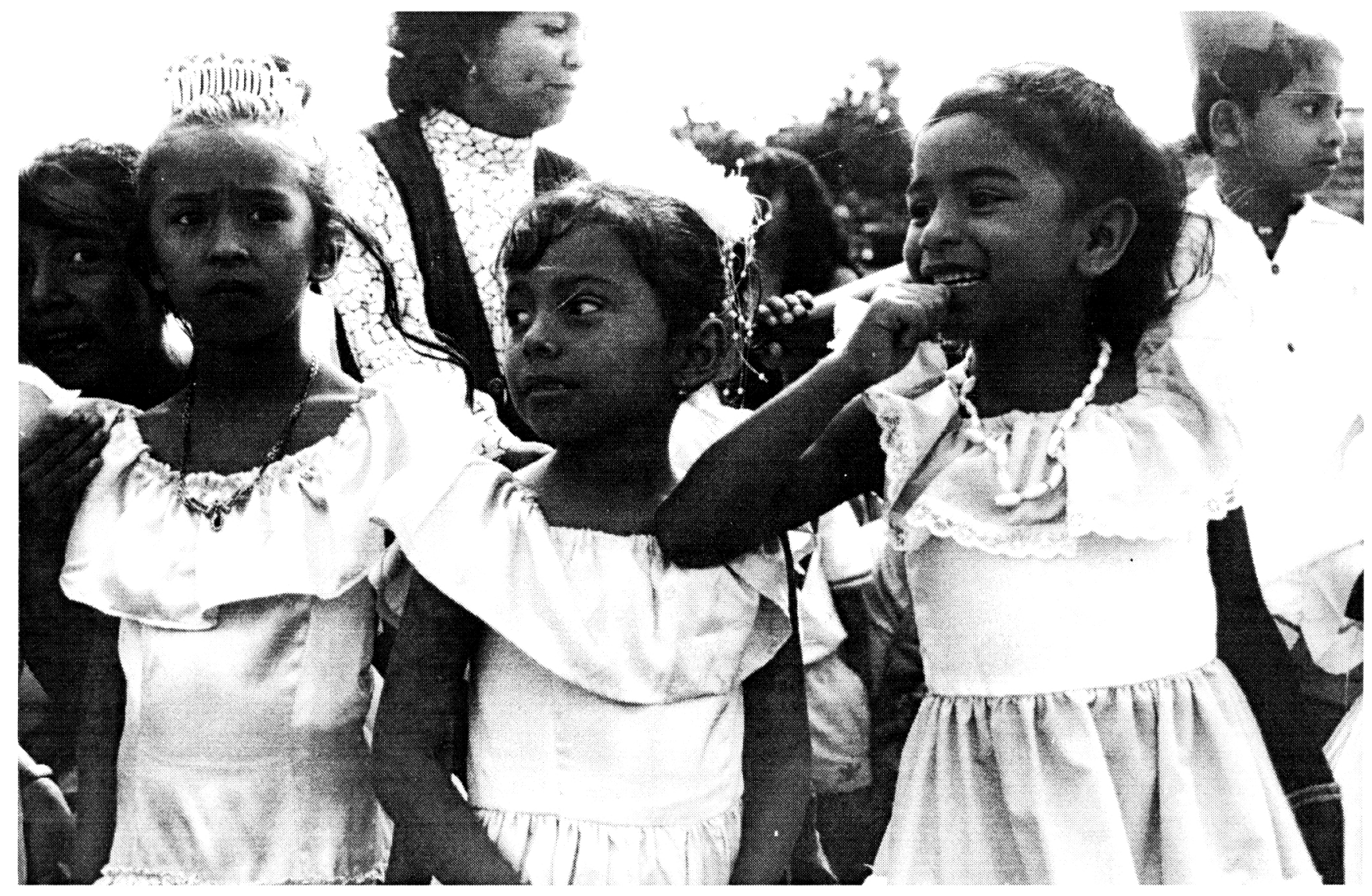




\section{NOVEDADES DE LA COLECCIÓN "CIENCIA POLÍTICA"}

Directores: Ismael Crespo y Pablo Oñate

\section{SISTEMAS POLÍTICOS DE LA UNIÓN EUROPEA}

Editado por Manuel Alcántara Sáez (Catedrático de Ciencia Política, Universidad de Salamanca), ISBN 84-8442-187-2, 559 páginas, 5900 pesetas.

En esta obra se estudian de forma sistemática los sistemas políticos de los países que constituyen la Unión Europea, así como las principales características de las instituciones y procesos políticos de la Unión. A partir de una estructura de análisis común, cada uno de los capítulos analiza los aspectos principales de los sistemas políticos, analizados por especialistas de cada caso concreto. Se estudian tanto sus aspectos formales (estructura del Estado, organización de poderes y relaciones entre ellos, y estructura política territorial), como los relativos al proceso y a los actores políticos (sistemas electorales y elecciones, partidos y sistemas de partidos, otros agentes políticos, cultura política, políticas públicas y las principales características socioeconómicas).

La descripción de los principales elementos y el análisis del funcionamiento de cada sistema político se apoya tanto en la revisión de la bibliografía más relevante de cada caso como en la utilización de una gran cantidad de datos e indicadores, que se incluyen en tablas y gráficos a lo largo de cada capítulo. Todo ello convierte al libro en una obra de referencia imprescindible para todo aquél que pretenda acercarse a la realidad política de los países de la Unión Europea y esta organización política misma.

\section{Contenido:}

Presentación (Manuel Alcantara Sáez)

Un capítulo dedicado a cada uno de los siguientes sistemas políticos:

- República Federal de Alemania (Raimundo Viejo Viñas)

- Austria (Carlos de Cueto)

- Bélgica (Mónica Méndez Lago)

- Dinamarca (Esther del Campo García)

- España (Pablo Oñate Rubalcaba)

- Finlandia (Esther del Campo García)

- Francia (Pilar Gangas Peiró)

- Grecia (Miguel Jerez Mir y José Real Dato)

- Holanda (Leticia M. Ruiz Rodríguez)

\author{
- Irlanda (Fátima García Díez y Araceli Mateos Díaz) \\ - Italia (Eva Anduiza Perea) \\ - Luxemburgo (Carmen Ortega Villodres) \\ - Portugal (Nieves Lagares) \\ - Reino Unido (Eva Anduiza Perea y Mónica Méndez Lago) \\ - Suecia (Esther del Campo García) \\ - El sistema político de la Unión Europea \\ (Carlos Closa y José Fernández Albertos)
}

\section{OTROS LIBROS DE LA COLECCIÓN CIENCIA POLITICA}

Cultura Política. Enfoques teóricos y análisis empíricos

Editores: Pilar del Castillo e Ismael Crespo (ISBN 84-8002-445-3, 2900 pesetas)

Política y gobierno en España

Editores: Manuel Alcántara y Antonia Martínez (ISBN 84-8002-528-X, 3900 pesetas)

Aspectos políticos y sociales de la integración europea

Editores: Ivan Llamazares y Fernando Reinares (ISBN 84-8002-749-5,2950 pesetas)

Política ambiental en España. Subsidiariedad y desarrollo sostenible

Editoras: Susana Aguilar, Nuria Font y Joan Subirats (ISBN 84-8002-897-1, 2900 pesetas)

Política y gobierno en los Estados Unidos (1945-1999)

Autor: Gustavo Palomares (ISBN 84-8002-962-5, 2500 pesetas)

Las elecciones al Parlamento Europeo, 1999

Editoras: Antonia Martínez y Mónica Méndez (ISBN 84-8442-0434, 3900 pesetas)

Ciencia de la Administración

Autores: Carles Ramió y Xavier Ballart (ISBN 84-8442-194-5, precio por determinar)

Formulario de pedido para enviar a la editorial (también disponible en http://www.tirant.com)

Nombre y apellidos/Institución:

NIF/CIF:

Dirección:

Población:

País:

Teléfono:

Provincia:

Código postal:

Fax

E-mail:

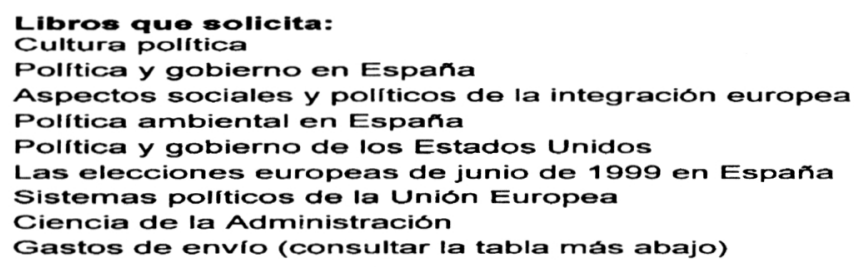

\section{Pesetas}

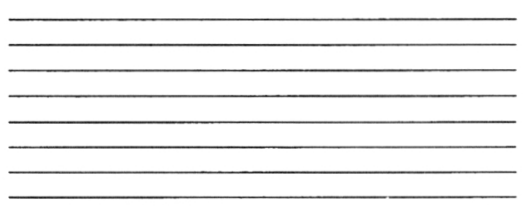

Total: 